\title{
A novel cardiovirus in wild rats
}

\author{
Yan Wang ${ }^{1 \dagger}$, Jing Zhao ${ }^{1 \dagger}$, Min Zheng ${ }^{2}$, Zhijian Liu', Wang Li', Xingli Fu', Yuan Lin', Jiaqi Yuan ${ }^{1}$, Jieji Zhao ${ }^{1}$, \\ Quan Shen ${ }^{1}$, Xiaochun Wang ${ }^{1}$, Hua Wang ${ }^{1}$ and Shixing Yang ${ }^{1 *}$ (D)
}

\begin{abstract}
Background: Cardioviruses cause severe illnesses in rodents and humans. In recent years, novel cardioviruses have been frequently found, which promoted further studies of the genetic diversity of cardioviruses. Using viral metagenomics, we genetically characterized a novel cardiovirus (named SX1) from wild rat feces. The genomic structure of SX1 shared similar features with those of the Theiler's murine encephalomyelitis viruses, including a leader protein, four structural proteins and seven non-structural proteins. Phylogenetic analysis based on both structural proteins and non-structural proteins coding regions showed that SX1 was formed into a separate branch, being located between the branches of Theiler's murine encephalomyelitis viruses and Thera viruses. Variable resides presented in the Ser/Thr rich domain of L protein, VP1 loops, and VP2 puffs distinguished SX1 from Theiler's murine encephalomyelitis viruses, suggesting the different antigenicity and pathogenicity of SX1.
\end{abstract}

Keywords: Cardiovirus genus, Viral metagenomics, Genomic structure, Phylogenetic analysis

\section{Findings}

Cardioviruses is a genus of picornaviruses that cause severe illnesses in rodents and human [1-4]. The genus Cardiovirus includes three species, namely Cardiovirus $A$, Cardiovirus B, and Cardiovirus C. Cardiovirus $A$ has only one member, encephalomycarditis virus (EMCV), which causes rat encephalitis and myocarditis [5, 6]; Cardiovirus $C$ is a novel cardiovirus identified in laboratory rats and Rattus norvegicus [7]; Cardiovirus $B$ is composed of Theiler's murine encephalomyelitis virus (TMEV), Vilyuisk human encephalomyelitis virus (VHEV) , Thera virus (TRV), and Saffold virus (SAFV), where VHEV and SAFV can infect humans and cause encephalomyelitis, acute gastroenteritis and so on [1, 8, 9]. TMEV and TRV mainly infect mice and cause neurological diseases $[3,10,11]$. TMEVs were originally isolated from colony-bred mice that developed spontaneous paralysis in the early 1930s [12]. Now it is reported that TMEVs mainly cause asymptomatic infections in mice, and in rare cases, neurological symptoms featured with early poliomyelitis or late demyelinating disease [13]. TRVs were firstly isolated from sentinel rats housed with TMEV-seropositive rats in Japan in 2002 [14]. In

\footnotetext{
* Correspondence: johnsonyang1979@163.com

${ }^{\dagger}$ Equal contributors

${ }^{1}$ School of Medicine, Jiangsu University, 301 Xuefu Road, Zhenjiang, Jiangsu 212013, People's Republic of China

Full list of author information is available at the end of the article
}

2008, a novel isolate of TRV was detected in the feces of rats [3]. Till now, there is no report about the association between TRV and diseases in rats. Using virus metagenomics method, we detected a novel cardiovirus in the feces of wild rats and characterized the complete genome. The novel cardiovirus was named SX1 and its genomic sequence was submitted to GenBank with accession no. MF172923.

In order to investigate rat intestinal virome, 40 intestinal content samples were collected from wild M. longicaudus rats captured by the Chinese Center for Disease Control and Prevention in Taizhou City from three districts of Taizhou City including Taixing $(n=15)$, Gaogang $(\mathrm{n}=15)$, and Hailing $(n=10)$ from June to August in 2014. All of the wild rats were adults and the exact ages were unknown.

Viral metagenomics method was used to identify viral sequences in these samples. Four separate pools were randomly generated, each of which contained 10 fecal specimens. Briefly, fecal samples were suspended in Dulbecco's phosphate-buffered saline (DPBS). After low speed centrifugation and filtration, the samples were treated with DNase and RNase to reduce levels of rat nucleic acids while viral genome within viral capsid was protected from digestion [15]. Four libraries were then constructed using Nextera XT DNA Sample Preparation Kit (Illumina) and sequenced using the Miseq Illumina 
platform with 250 bases paired ends with a distinct molecular tag for each pool. Bioinformatics analysis was performed according to a previous study [15].

Miseq Illumina sequencing generated 16,832 unique reads which contained abundant viral sequences reads based on BLASTx search. Numbers of sequence reads showing similarities to known viruses included 2097 in pool-1, 5375 unique reads in pool-2, 7953 reads in pool-3, and 1407 reads in pool-4. Pool-3 contained 417 sequence reads showed significant sequence similarities to Theilovirus. PCR was then performed to bridge the gaps between sequence contigs and Rapid Amplification of cDNA Ends (RACE) amplification was used to acquire the complete genome. The resulted complete genome of SX1 is consisted of $8102 \mathrm{bp}$, in addition to a poly (A) at $3^{\prime}$ terminus. A single 2304-amino acid polyprotein is found which is composed by 12 proteins, including a leader peptide (L), four structural proteins (VP1, VP2, VP3, and VP4), and seven nonstructural proteins (2A, 2B, 2C, 3A, $3 \mathrm{~B}, 3 \mathrm{C}$, and 3D). Using the SimPlot software [16], the sequence similarity and putative homologous recombination analyses between SX1 and the other strains of Cardiovirus $B$ were performed. Results showed that SX1 had higher sequence similarity to TMEVs than TRVs and SAFVs (Fig. 1).
In order to further determine the genetic relationship of SX1 with the other members of Cardioviruses, phylogenetic analysis based on both the structural protein region (P1) and the nonstructural protein regions (P2, and P3) showed that SX1 showed that SX1 formed a separate branch (Fig. 2a and b). Sequence comparison between SX1 and TMEVs, TRVs, SAFV, and VHEV indicated the amino acid sequence identities of P1 and P2 + P3 were $63-85 \%$ and $48-84 \%$, respectively. The P1 region of SX1 shared the highest amino acid sequence identity (85\%) with a TRV strain (GenBank accession no. EU542581), which was isolated from infected rats [3]. The P2 + P3 region of SX1 shared the highest amino acids sequence identity $(84 \%)$ with a TMEV strain (GenBank assession no. KJ191558), which was isolated from the wild boars. Based on the VP1 region, SX1 shared the amino acid sequence identities $<77.9 \%$ with TMEVs and TRVs, while the identities among the members within TMEVs and TRVs were 87.7-94.6\%, 92.7-97.1\%, respectively. Although SX1 failed to meet the criteria that a new Cardiovirus species should have $<60 \%$ aa identity of P1 comparing with the other known Cardiovirus species according to the International Committee on Taxonomy of Viruses, the results of

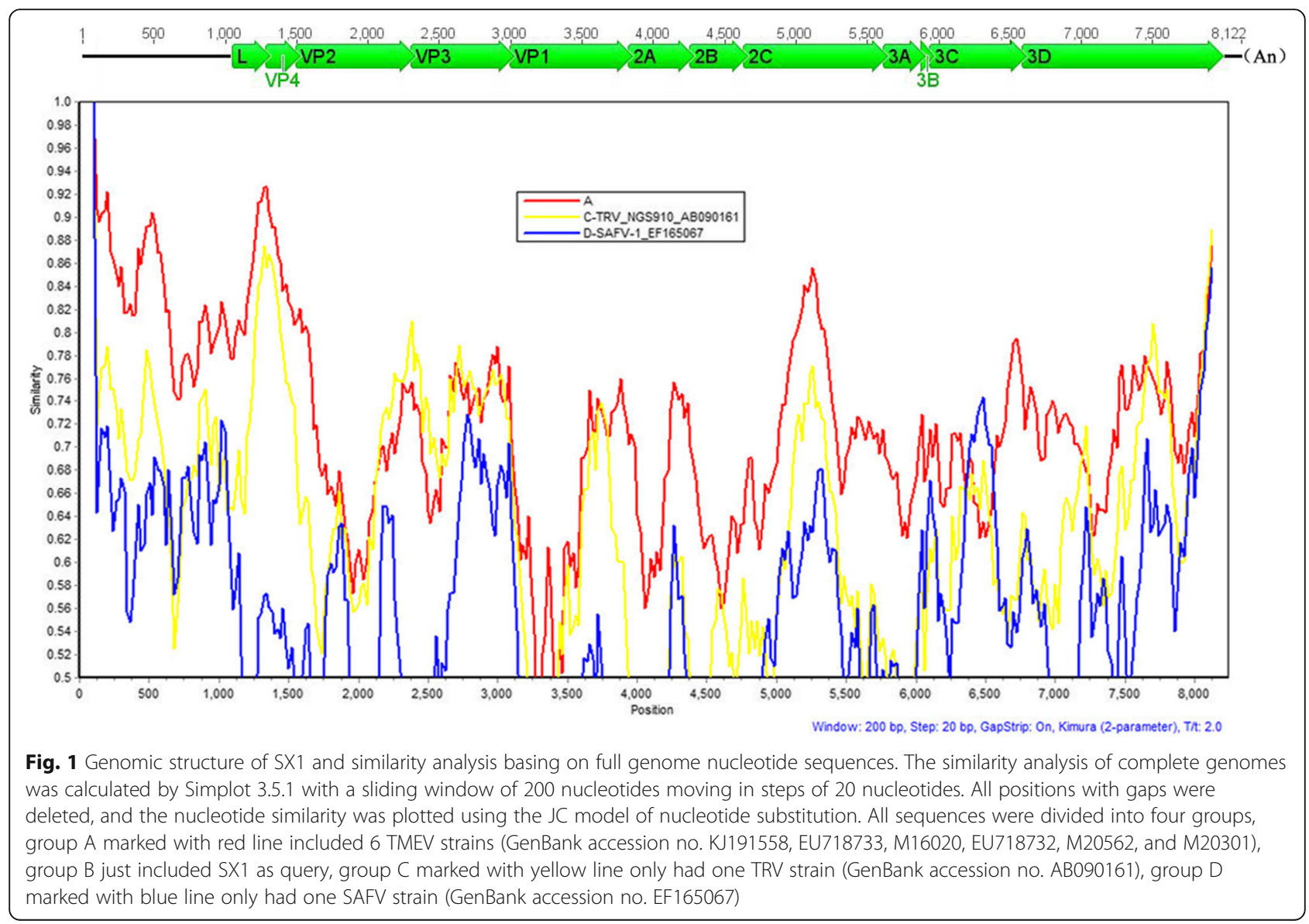




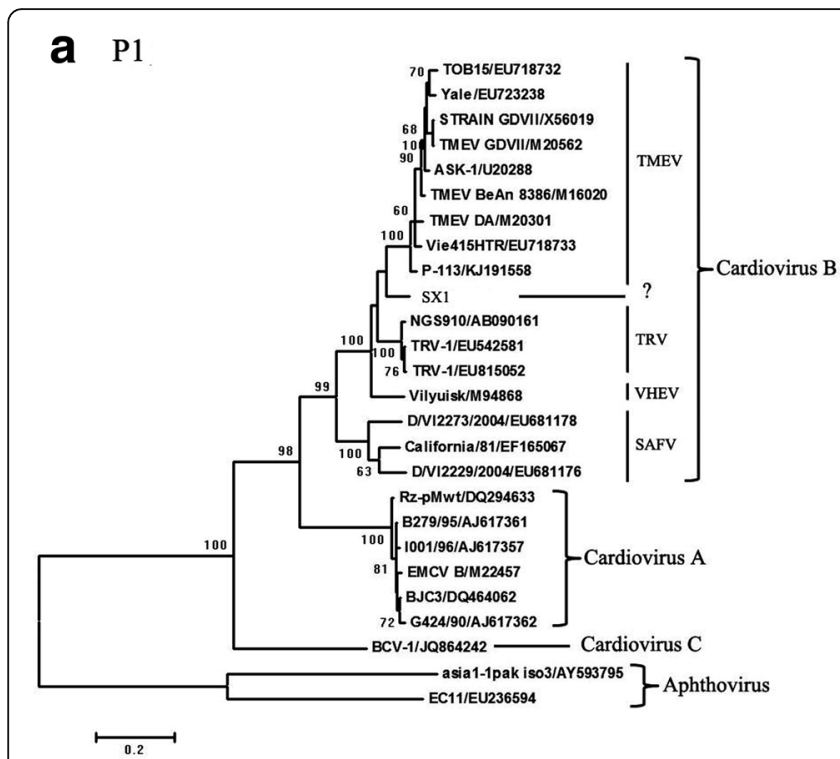

\section{b $\quad \mathrm{P} 2+\mathrm{P} 3$}

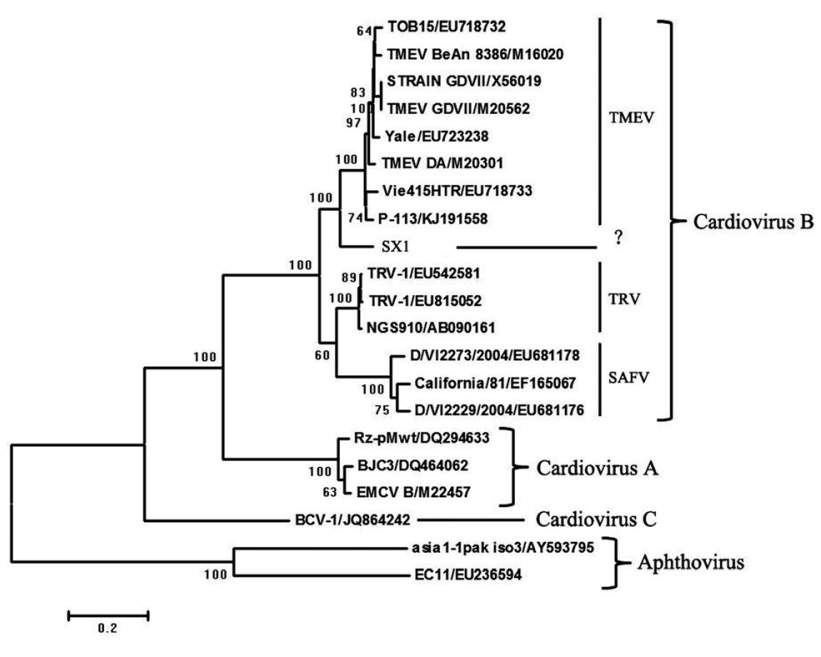

Fig. 2 Phylogenetic relationships among cardioviruses based on the alignment of the nucleotide sequences of the P1, and P2 + P3 coding regions. a Phylogenetic analysis based on the P1 region. b Phylogenetic analysis based on the P2 and P3 regions. An unrooted tree was reconstructed using the neighbor-joining algorithm implemented in MEGA 6.0. Strain names and GenBank accession numbers of cardioviruses were shown. Scale bar indicated nucleotide substitution per site. Bootstrap valued (based on 1000 replicates) for each node are given

phylogenetic analysis indicated that SX1 might be a novel type of Cardiovirus.

We further analyzed the key proteins of SX1 which mainly related to antagonizing host immunity or being involved in host cell tropism and viral pathogenesis, including L protein [17], VP2 puffs $A$ and B, and VP1 loops I and II [18]. The L protein is thought to have multiple functions including the regulation of the trafficking of interferon-regulatory proteins to the nucleus, binding to Ran GTPase and blocking the nuclear export of new mRNAs $[17,19]$. Being similar to TMEV, SX1 encoded an L protein including three domains: an N-terminal atypical (CHCC) zinc finger, an acidic domain, and a C-terminal Ser/Thr-rich domain (Fig. 3a). Compared with other TMEVs, there were two mutations of Ser and Thr residues in the Ser/ Thr-rich domain of SX1 (Fig. 3a), which is associated with the viral pathogenicity. Whether the mutations can affect viral pathogenicity remains to be addressed. In addition, a 156-aa $L^{*}$ protein was also encoded 13 nucleotides downstream of the start site of polyprotein (data not shown), which was thought to have a function in viral persistence because the mutant leading to early termination of $\mathrm{L}^{*}$ protein inhibited TMEV persistence [20]. Comparing with other TMEVs and VHEVs, L* protein of SX1 shared the highest amino acid sequence identity (79\%) with one VHEV strain which caused degenerative neurological disease of inhabitants of Siberia [21]. In cardioviruses, the surface of VP1 loops I and II, VP2 puffs A and B are exposed on the capsid surface and are thought to be involved in host cell tropism and viral pathogenesis [18]. Comparing with TMEVs and TRV NGS910 strain, although VP1 loop I of SX1 was more similar to TMEVs, VP1 loop II, VP2 puffs A and B of SX1 had higher amino acid sequence identities with TRV NGS910 strain (Fig. 3b and c). TMEVs VP2 puff B is believed to be responsible for sialic acid binding for viral entry, where three VP2 amino acids of puff B $\left(\mathrm{Q}^{2161}, \mathrm{~A}^{2163}\right.$, and $\left.\mathrm{G}^{2174}\right)$ within a positively charged area on the viral surface are vital for this binding [22]. Comparing with TMEVs, only two of the three residues in SX1 were the same to TMEVs (Fig. 3c). These data support our preceding conclusion in the phylogenetic analysis that SX1 is a novel strain with different epitopes and may have different pathogenicity.

To investigate the prevalence of SX1 in rats, we designed nested PCR primers targeting VP2 gene of cardiovirus by RT-PCR. Primer SX1 F1 (5'-GCCCATCGCGG AGAACACCC-3') and SX1 R1 (5'-TGTCCAGGAGCT GGTCGGGG-3') were used for the first round of PCR, and SX1 F2 (5'-CGGGGCTTTCTCCCACGTTCG-3') and SX2 R2 (5' - CGTTTCGGCCGTCATAGCGGT-3') for the second round, the expected length of amplicons was $308 \mathrm{bp}$. PCR screening results showed that 3 of the 40 fecal samples were positive with positive rate of $7.5 \%$ (3/40). The three positive samples were all obtained from rats collected in Gaogang. The specific PCR products were sequenced by Sanger method. Sequence alignment showed that the three 308 bp sequences shared > 


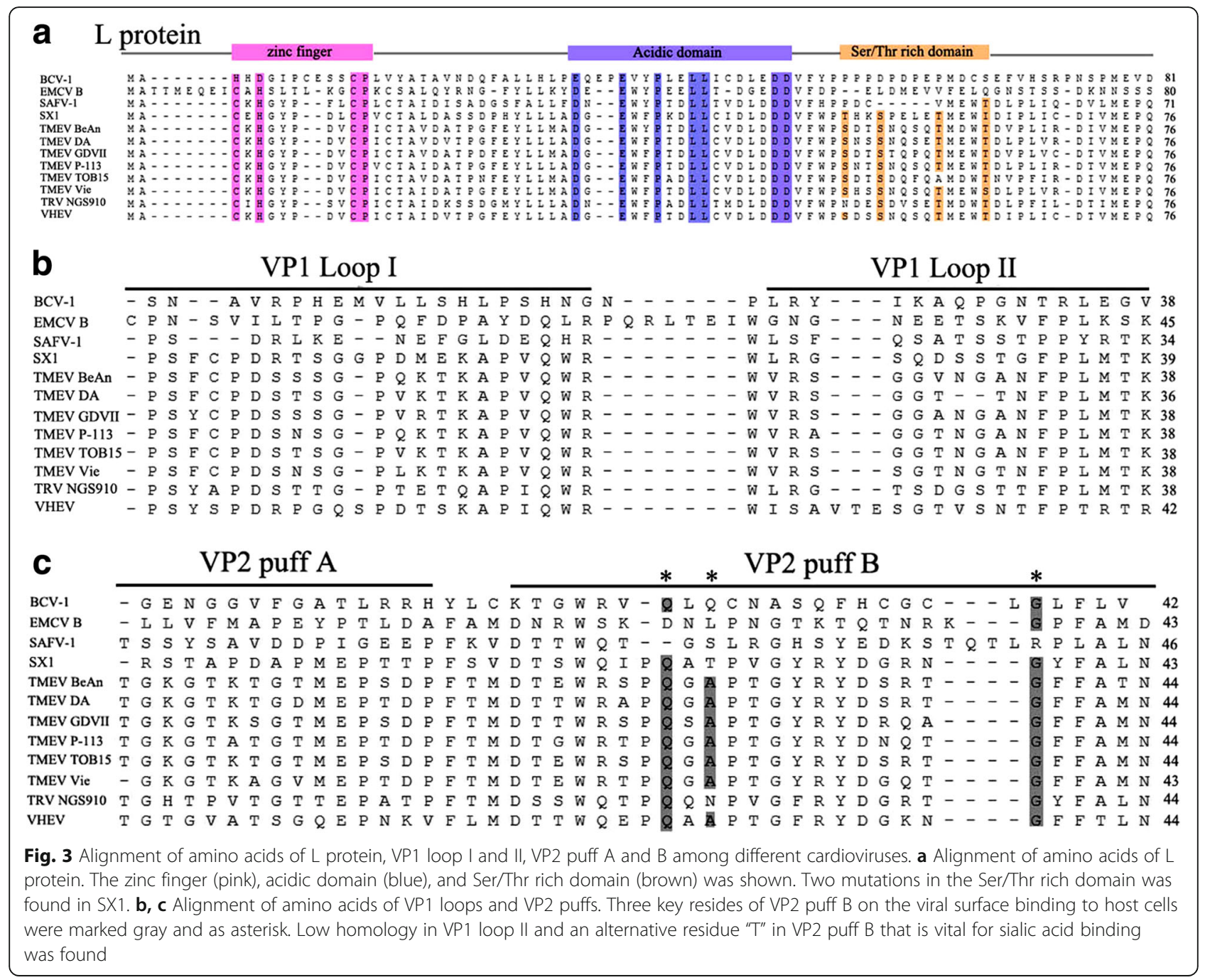

98\% nucleotide identities with each other, while shared $<79 \%$ nucleotide identities with other members of the genus, indicating the prevalence of a single strain in the wild rats in this area.

\section{Conclusions}

In summary, we identified a novel cardiovirus in wild rats and characterized its complete genome. SX1 encodes a polyprotein including an $\mathrm{L}$ protein, four structural proteins and seven non-structural proteins, in addition, an $L^{*}$ protein is encoded 13 nucleotides downstream of the start site. Comparing with TMEVs and TRVs, many amino acids are different among them in loops of VP1 and puffs of VP2, both of those domains are vital for viral binding with host cells. The amino acid differences in those domains indicate that SX1 may have different pathogenic and antigenic properties. Phylogenetic analysis showed that the SX1 formed a separate branch that was distant from TMEVs and TRVs. The epidemiologic study suggested a single strain was prevalent in the wild rats in this area.

\section{Abbreviations}

EMCV: Encephalomycarditis virus; SAFV: Saffold virus; TMEV: Theiler's murine encephalomyelitis virus; TRV: Thera virus; VHEV: Vilyuisk human encephalomyelitis virus

\section{Acknowledgements}

We thank Taizhou Center for Disease Control and Prevention to samples collection. We thank Dr. Yanjin Zhang for helpful comments on this manuscript.

\section{Funding}

This work was partly supported by the National Key Research and Development Programs of China No. 2017YFC1200201, the Natural Science Foundation of Jiangsu Province No. BK20140537 and BK20140578, the National Natural Science Foundation of China No. 31402211, the Professional Research Foundation for Advanced Talents of Jiangsu University No. 12JDG085 and 13JDG087, China Postdoctoral Foundation No. 2014 M561597, and Jiangsu Postdoctoral Foundation No. 1302057C.

\section{Availability of data and materials}

All relevant data are included in the manuscript. Nucleotide sequences are available at the GenBank depository under accession numbers: MF172923MF192925. 


\section{Authors' contributions}

YW and SY conceived the study. All authors participated in the experiments. SY wrote the paper. All authors read and approved the final manuscript.

\section{Ethics approval and consent to participate}

Not applicable.

\section{Consent for publication}

Not applicable.

\section{Competing interests}

The authors declare that they have no competing interests.

\section{Publisher's Note}

Springer Nature remains neutral with regard to jurisdictional claims in published maps and institutional affiliations.

\section{Author details}

${ }^{1}$ School of Medicine, Jiangsu University, 301 Xuefu Road, Zhenjiang, Jiangsu 212013, People's Republic of China. ²Department of Gynecology, Rizhao Maternity\& Infant Health Hospital, Rizhao, Shandong 276800, People's Republic of China. ${ }^{3}$ School of Basic Medical Sciences, Ningxia Medical University, Yinchuan, Ningxia 750000, People's Republic of China.

Received: 6 June 2017 Accepted: 19 March 2018

Published online: 27 March 2018

\section{References}

1. Drexler JF, Luna LK, Stocker A, Almeida PS, Ribeiro TC, Petersen N, Herzog P, Pedroso C, Huppertz HI, Ribeiro Hda C Jr, et al. Circulation of 3 lineages of a novel Saffold cardiovirus in humans. Emerg Infect Dis. 2008;14:1398-405.

2. Chiu CY, Greninger AL, Chen EC, Haggerty TD, Parsonnet J, Delwart E, Derisi $J$, Ganem D. Cultivation and serological characterization of a human Theiler's-like cardiovirus associated with diarrheal disease. J Virol. 2010;84: 4407-14.

3. Drake MT, Riley LK, Livingston RS. Differential susceptibility of SD and CD rats to a novel rat theilovirus. Comp Med. 2008;58:458-64.

4. Blinkova O, Kapoor A, Victoria J, Jones M, Wolfe N, Naeem A, Shaukat S, Sharif S, Alam MM, Angez M, et al. Cardioviruses are genetically diverse and cause common enteric infections in south Asian children. J Virol. 2009;83: 4631-41.

5. Hill BD, Ketterer PJ, Rodwell BJ, Eaves FW, Webster WR. Encephalomyocarditis virus infection and pig disease in Queensland. Aust Vet J. 1985;62:433-4

6. Philipps A, Dauber M, Groth M, Schirrmeier H, Platzer M, Krumbholz A Wutzler P, Zell R. Isolation and molecular characterization of a second serotype of the encephalomyocarditis virus. Vet Microbiol. 2012;161:49-57.

7. Hansen TA, Mollerup S, Nguyen NP, White NE, Coghlan M, Alquezar-Planas $\mathrm{DE}$, Joshi $\mathrm{T}$, Jensen $\mathrm{RH}$, Fridholm $\mathrm{H}$, Kjartansdottir $\mathrm{KR}$, et al. High diversity of picornaviruses in rats from different continents revealed by deep sequencing. Emerg Microbes Infect. 2016;5:e90.

8. Lipton HL. Human Vilyuisk encephalitis. Rev Med Virol. 2008;18:347-52

9. Liang Z, Kumar AS, Jones MS, Knowles NJ, Lipton HL. Phylogenetic analysis of the species Theilovirus: emerging murine and human pathogens. J Virol. 2008;82:11545-54

10. Woodland DL. Theiler's murine encephalomyelitis virus. Viral Immunol. 2017; 30(5):303

11. Carrillo-Salinas FJ, Mestre L, Mecha M, Feliu A, Del Campo R, Villarrubia N, Espejo C, Montalban X, Alvarez-Cermeno JC, Villar LM, Guaza C. Gut dysbiosis and neuroimmune responses to brain infection with Theiler's murine encephalomyelitis virus. Sci Rep. 2017;7:44377.

12. Theiler M. Spontaneous encephalomyelitis of mice-a new virus disease. Science. 1934:80:122.

13. Lehrich JR, Arnason BG, Hochberg FH. Demyelinative myelopathy in mice induced by the DA virus. J Neurol Sci. 1976:29:149-60.

14. Ohsawa K, Watanabe $Y$, Miyata H, Sato H. Genetic analysis of a Theiler-like virus isolated from rats. Comp Med. 2003:53:191-6.

15. Zhang W, Yang S, Shan T, Hou R, Liu Z, Li W, Guo L, Wang Y, Chen P, Wang $X$, et al. Virome comparisons in wild-diseased and healthy captive giant pandas. Microbiome. 2017;5:90.
16. Lole KS, Bollinger RC, Paranjape RS, Gadkari D, Kulkarni SS, Novak NG, Ingersoll R, Sheppard HW, Ray SC. Full-length human immunodeficiency virus type 1 genomes from subtype C-infected seroconverters in India, with evidence of intersubtype recombination. J Virol. 1999;73:152-60.

17. Kong WP, Ghadge GD, Roos RP. Involvement of cardiovirus leader in host cell-restricted virus expression. Proc Natl Acad Sci U S A. 1994:91:1796-800.

18. Inaoui K, Michiels T. Adaptation of Theiler's virus to L929 cells: mutations in the putative receptor binding site on the capsid map to neutralization sites and modulate viral persistence. Virology. 1998;244:397-404.

19. Porter FW, Bochkov YA, Albee AJ, Wiese C, Palmenberg AC. A picornavirus protein interacts with ran-GTPase and disrupts nucleocytoplasmic transport. Proc Natl Acad Sci U S A. 2006;103:12417-22.

20. Chen HH, Kong WP, Zhang L, Ward PL, Roos RP. A picornaviral protein synthesized out of frame with the polyprotein plays a key role in a virusinduced immune-mediated demyelinating disease. Nat Med. 1995:1:927-31.

21. Pritchard AE, Strom T, Lipton HL. Nucleotide-sequence identifies Vilyuisk virus as a divergent Theiler virus. Virology. 1992;191:469-72.

22. Zhou L, Luo Y, Wu Y, Tsao J, Luo M. Sialylation of the host receptor may modulate entry of demyelinating persistent Theiler's virus. J Virol. 2000;74 $1477-85$

\section{Submit your next manuscript to BioMed Central and we will help you at every step:}

- We accept pre-submission inquiries

- Our selector tool helps you to find the most relevant journal

- We provide round the clock customer support

- Convenient online submission

- Thorough peer review

- Inclusion in PubMed and all major indexing services

- Maximum visibility for your research

Submit your manuscript at www.biomedcentral.com/submit
Biomed Central 